\title{
Analysis of a New Hidden Attractor Coupled Chaotic System and Application of Its Weak Signal Detection
}

\author{
Wenhui Luo ${ }^{1},{ }^{1}$ Qingli Ou, ${ }^{1}$ Fei Yu, ${ }^{2}$ Li Cui, ${ }^{1}$ and Jie Jin ${ }^{1,3}$ \\ ${ }^{1}$ Hunan University of Science and Technology, Xiangtan 411201, Hunan, China \\ ${ }^{2}$ School of Computer and Communication Engineering, Changsha University of Science and Technology, \\ Changsha 410114, China \\ ${ }^{3}$ College of Information Science and Engineering, Jishou University, Jishou 416000, China \\ Correspondence should be addressed to Wenhui Luo; wenhuiluo@mail.hnust.edu.cn
}

Received 1 October 2020; Revised 4 November 2020; Accepted 10 December 2020; Published 23 December 2020

Academic Editor: Viet-Thanh Pham

Copyright (c) 2020 Wenhui Luo et al. This is an open access article distributed under the Creative Commons Attribution License, which permits unrestricted use, distribution, and reproduction in any medium, provided the original work is properly cited.

\begin{abstract}
In order to improve the complexity of the chaotic system and the accuracy of the weak signal detection, this paper propose a new hidden attractor coupled chaotic system and a corresponding weak signal detection system, which can be used to obtain the phase diagram of the proposed system using the fourth order of the Runge-Kutta method. The dynamic behavior of the chaotic system is analyzed through the bifurcation diagram, Lyapunov exponent, and power spectrum. The Lyapunov exponent is used to depict the basins of attraction for the system. After research, it is discovered that symmetry exists in the system. Comparative analysis has demonstrated that the system has higher detection accuracy and excellent antinoise performance. Finally, the circuit simulation and FPGA realization of the system indicated that the numerical simulation results are consistent with the FPGA implementation results, proving the theoretical analysis to be correct and the accuracy of the detection results.
\end{abstract}

\section{Introduction}

After Lorenz introduced the Lorenz system [1] in 1963, the chaos theory has become a hot spot of nonlinear field research. With the deepening of theoretical research, researchers have conducted a lot of analysis into the attractor and basins of attraction of the chaotic system. In [2], after an investigation into the basins of attraction of coexistence attractors in the coupled Duffing system, the riddled basins of attraction of the Chen system are studied, and the riddled basins of attraction have a positive repulsive set of Lebesgue measures via mathematical proofs. It is found in [3] that the number of coexisting attractors is different when the initial values are different. A numerical method for estimating the shortening calculation time of the relative area of the basins of attraction is proposed in [4], and its application scope is verified. In [5], the mixed basins of attraction formed when multiple attractors coexist in a chaotic system are analyzed, and the research shows that the basins of attraction have a fractal regular structure. In addition, the hidden attractors [6] and hidden bifurcations in the system have also attracted extensive attention of researchers. For example, the phenomenon of hidden attractor and hidden bifurcation in multiscroll Chua chaotic system is studied in [7]. The dynamic characteristics of multistable chaotic systems with hidden attractors are studied in [8]. A four-wing hidden attractor chaos is studied in [9]. In [10], a threedimensional hidden attractor chaotic system with Lyapunov dimension of 2.9075 is studied, and a Bluetooth device for autonomous wireless mobile robot based on chaotic motion controller is proposed. At the same time, as a special state of chaotic system, multistability has been widely studied. The chaotic system with multiple attractors coexisting and multistability are analyzed in $[11,12]$. Reference [13] analyzed the multistability of a chaotic system with two circles of equilibrium points and implemented the system using FPGA technology. With the research of electronic components, the realization of memristors provides a new method for the construction of 
chaotic systems. Nowadays, chaotic systems based on memristors [14-16] and chaotic neural networks [17-19] have received great attention from researchers.

With the in-depth theoretical research of chaotic systems, their engineering application has become more mature. In recent years, the application of chaotic systems has spread throughout many fields including electronic circuit [20-24], image processing [25-30], secure communication [31-34], complex networks [35-38], and random signal generation [39-41]. Traditional stochastic resonance [42], wavelet transform [43], and empirical mode decomposition [44] are widely used in early fault signal detection. The stochastic resonance method enhances the signal utilizing noise, destroying the original information of the signal. Immune to certain noises and sensitive to specific signals, the chaotic system is featured by simple detection methods and retention of the original signal information. In 1992, Birx and Pipenberg first proposed detecting weak signals by using chaotic oscillators [45], and, since then, the chaotic systems have been represented by the Duffing system and the van der Pol system [46, 47] began to be widely applied in weak signal detection. This research, based on the time domain and frequency domain detection methods for weak signals [48, 49] and the antinoise of chaotic system [50], enables chaotic system detection methods to be more mature. The criteria for judging the state of chaotic systems will directly affect the accuracy of detection; thus, scholars have also conducted in-depth studies on the calculation of the threshold of chaotic systems. In [51], Melnikov method is used to identify chaotic features. In [52], the threshold of the system is determined by solving the Lyapunov exponent. At present, most of the researches on weak signals based on chaos theory use a single chaotic system for detection. The system proposed in [53] can only detect signals with a size of 0.01 and the SNR can only reach $-45.85 \mathrm{~dB}$. The system in [54] can detect signals with a size of $1 \times 10^{-9}$, but a noise signal that can be immune to it can only reach the order of $1 \times 10^{-9}$. The system in [55] can detect a signal with a wider frequency range, but the detection range of the initial phase angle of the signal is too large and thus is unfavorable for determining the signal parameters. At present, most of the researches on weak signals based on chaos theory use a single chaotic system for detection. Compared with the single chaotic system, the coupled system has excellent stability, high antinoise performance, and low detection threshold. Therefore, the coupled chaotic system has high theoretical research value and practical application value.

To improve the chaotic system complexity and the accuracy of signal detection, this paper proposes a new hidden attractor coupled chaotic system. Through an analysis of the system's phase diagram, the Lyapunov index, and so forth, the complex dynamic behavior of the system is explained in detail. Moreover, the basins of attraction for the system are drawn using the Lyapunov index to study the characteristics of the system's attractor. Theoretical analysis indicates that the system is sensitive to initial values and immune to noise, and the coupled chaotic system is applied to weak signal detection. Finally, through OrCAD circuit simulation and FPGA implementation, the correctness of the theoretical analysis and the accuracy of signal detection are verified. Through data analysis and experimental data, the system proposed in this paper can detect signals sized $1 \times 10^{-6}$, and the signal-to-noise ratio reaches $-73.892 \mathrm{~dB}$. Moreover, the immune signal reached the order of $3.5 \times 10^{-3}$, and the error range of the initial phase angle is \pm 0.09 . Therefore, the detection performance of the coupled system proposed in this paper is better than the single chaotic system proposed in $[45,46,53-55]$.

\section{Coupled Chaotic System Model and Dynamic Analysis}

2.1. Coupled Chaotic System Model. Through [45-47], a single chaotic system with different nonlinear terms is analyzed, and the limitations of signal detection performance detection are studied at the same time. For example, [53] can detect low signal amplitude, and [54] can be immune. The noise is small, and [55] detects that the initial phase angle error of the signal is too large. Based on the above reasons, this paper proposes a new system:

$$
\left\{\begin{array}{l}
\ddot{x}+c_{1} \dot{x}-x+c_{2} x^{5}+k(x-y)=f \cos (\omega t+\theta) \\
\ddot{y}+c_{3}\left(1-y^{3}\right) \dot{y}+y+y^{3}+k(y-x)=f \cos (\omega t+\theta)
\end{array}\right.
$$

where $k$ is the linear coupling coefficient of system (1). The larger the coefficient, the higher the degree of coupling; $c 1, c 2$, and $c 3$, respectively, represent the coefficients of the nonlinear terms. $f \cos (\omega t+\theta)$ is the driving force of the system, and $f, \omega$, and $\theta$ represent the amplitude, frequency, and initial phase angle of the driving force, respectively.

2.2. Analysis of the Equilibrium Point and Lyapunov Exponent of a Coupled Chaotic System. We obtained system (2) by coupling system (1) and taking the coefficients $c 1=0.5, c 2=0.2, c 3=0.8$, and $k=0.4$, with the state space model expression of system (2) shown in the following equation:

$$
\left\{\begin{array}{l}
\dot{x}=z \\
\dot{y}=q \\
\dot{z}=-c_{1} z+x-c_{2} x^{5}-k(x-y)+f \cos (\omega t+\theta) \\
\dot{q}=-c_{3}\left(1-y^{2}\right) q-y-y^{3}-k(y-x)+f \cos (\omega t+\theta)
\end{array}\right.
$$

To simulate the coupled chaotic system (2), take the frequency $\omega=1$ and the initial phase angle $\theta=0$. The system's initial value is $(-1,-1,0,0)$. When the magnitude $f$ of the driving force takes $0.3,0.46,0.8$, and 1.2 , the homoclinic orbit, the period-doubling bifurcation, chaotic state, and periodic state will appear in system (2). The driving signal of system (2) is defined as $H$, and the signal as a whole system (2) is analyzed. Let the left side of the equation be equal to 0 ; then, 


$$
\left\{\begin{array}{l}
z=0 \\
q=0 \\
-0.5 z+x-0.2 x^{5}-0.4(x-y)+H=0 \\
-0.8\left(1-y^{2}\right) q-y-y^{3}-0.4(y-x)+H=0 .
\end{array}\right.
$$

When $H=1$, equation (3) has a real number solution $x 1=1.6299, y 1=0.8060, z 1=q 1=0$, and fourteen complex number solutions. With the changes of $H$, equation (3) will have an infinite number of equilibrium points, so system (2) is a hidden attractor coupled chaotic system. The Jacobian matrix of system (2) at the equilibrium point $M\left(x_{1}, y_{1}, z_{1}, q_{1}\right)$ is

$$
J=\left[\begin{array}{cccc}
0 & 0 & 1 & 0 \\
0 & 0 & 0 & 1 \\
1-x_{1}^{4}-0.4 & 0.4 & -0.5 & 0 \\
0.4 & -1.4-3 y_{1}^{2} & 0 & -0.8\left(1-y_{1}^{2}\right)
\end{array}\right] .
$$

Let $|J-\lambda I|=0$; its characteristic values $\lambda_{1}=-0.2486$ $+2.5373 i, \quad \lambda_{1}=-0.2486-2.5373 i, \quad \lambda_{3}=-0.1415+1.6983 i$, and $\lambda_{4}=-0.1415-1.6983 i$ can be obtained. According to the Routh-Hurwitz criterion, the real parts of the eigenvalues are all less than 0 , so system (2) is stable at the equilibrium point $\left(x_{1}, y_{1}, z_{1}, q_{1}\right)$. When $H=1$, the waveform diagram of each state variable phase changing over time and phase diagram within system (2) are shown in Figure 1.

Derivation of equation (2) can achieve

$$
\begin{aligned}
\nabla V & =\frac{\partial \dot{x}}{\partial x}+\frac{\partial \dot{y}}{\partial y}+\frac{\partial \dot{z}}{\partial z}+\frac{\partial \dot{q}}{\partial q} \\
& =-1.3+0.8 y^{2} .
\end{aligned}
$$

When $y \in[-1.2748,1.2748], \nabla V<0$, and system (2) is a dissipative system.

Let $\beta=\omega t$ and take $\omega=1$ and $\theta=0$. Convert system (2) into the form shown in the following equation:

$$
\left\{\begin{array}{l}
\dot{x}=z, \\
\dot{y}=q, \\
\dot{z}=-0.5 z+x-0.2 x^{5}-0.4(x-y)+f \cos \beta, \\
\dot{q}=-0.8\left(1-y^{2}\right) q-y-y^{3}-0.4(y-x)+f \cos \beta, \\
\dot{\beta}=1,
\end{array}\right.
$$

and the previous equation can be expressed by vector method as

$$
\dot{X}(t)=w(X(t) ; \eta) .
$$

The state space variable $X(t)=[x(t), y(t), z(t)$, $q(t), \beta(t)]^{T}, \quad w=\left[w_{1}, w_{2}, w_{3}, w_{4}, w_{5}\right]^{T}, \quad \eta$ represents the parameter set, and $[\cdots]^{T}$ represents the transposed matrix. The deviation $\delta X$ equation from orbit $\mathrm{X}(\mathrm{t})$ can be expressed as $\delta \dot{X}(t)=L_{i j}(X(t) ; \eta) \delta X, i, j=1,2, \ldots, 5 . L_{i j}=\left(\partial w_{i} / \partial x_{j}\right)$ and the Jacobian matrix form is

$$
\left.\begin{array}{ccc}
1 & 0 & 0 \\
0 & 1 & 0 \\
-0.5 & 0 & -f \sin \beta \\
0 & -0.8\left(1-y^{2}\right) & -f \sin \beta \\
0 & 0 & 0
\end{array}\right]
$$

When $f=0.8$, the Lyapunov exponents are $L_{E 1}=0.184134, L_{E 2}=0, L_{E 3}=-0.305903, L_{E 4}=-0.312796$, and $L_{E 5}=-0.697360$, respectively. The value of $L_{E 1}$ is greater than 0 , and system (2) is in a chaotic state. The sum of Lyapunov exponents is less than 0 , and system (2) is in a dissipative system, which is consistent with the previous results. In a chaotic state, the phase diagram of system (2) and the cross section diagram of Poincare are shown in Figure 2.

\section{Bifurcation of the Driving Force Parameters and Lyapunov Exponent Spectrum Analysis}

As the various parameters of the driving force have a very significant impact on the dynamics of the system, focus on the analysis of the parameters $f, \omega$, and $\theta$ was implemented, and the initial values of system (2) take $(-1,-1,0,0)$ on a uniform basis. When $\omega=1, \theta=0$. When the amplitude $f$ changes within the interval $[0,2]$, the bifurcation diagram and Lyapunov exponent spectrum of system (2) are shown in the following diagrams $3(\mathrm{a})$ and $3(\mathrm{~b})$. As shown in Figure 3(a), system (2) enters chaos from the period-doubling bifurcation, the chaotic state interval is approximately $[0.41,0.88]$, and the system is in a periodic state in the subsequent interval. According to the comparison between Figures $3(a)$ and $3(b)$, the intervals of the chaotic and periodic states of the system are consistent.

When $f=0.8, \theta=0$, and when the frequency $\omega$ changes in the interval $[0,3]$, the bifurcation diagram and Lyapunov exponent spectrum of system (2) are shown in Figures 4(a) and 4(b). As shown in Figure 4(a), the system enters a chaotic state from a period-doubling bifurcation. As seen from Figures 4(a) and 4(b), the change of frequency $\omega$ has a more significant impact on the system than the amplitude $f$. The bifurcation diagram of $\omega$ in the interval in $[0,0.8]$ and $[1,2]$ is shown in Figures 4(c) and 4(d). As seen from the 


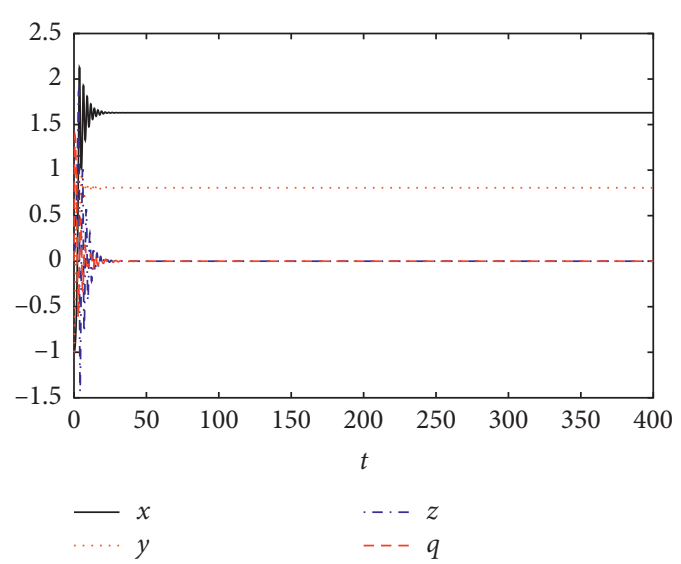

(a)

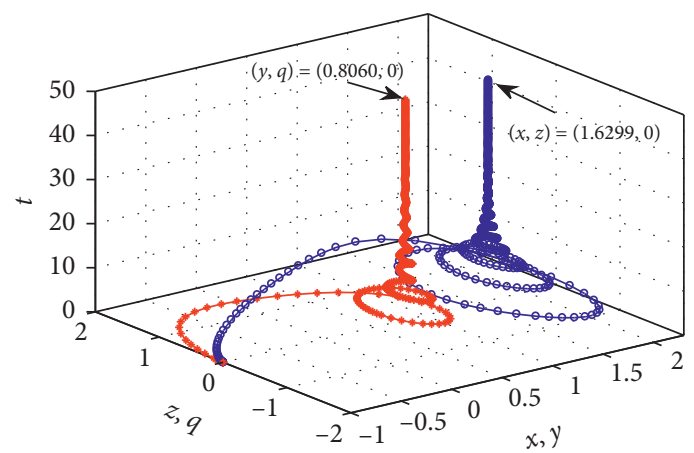

$\rightarrow x-z$

(b)

Figure 1: (a) When $H=1$, system (2) state variables are $x,(y) z, q$, and the waveform diagram. (b) When (H) $=1$, system (2) $x-y$ and $z-q$ phase diagram.

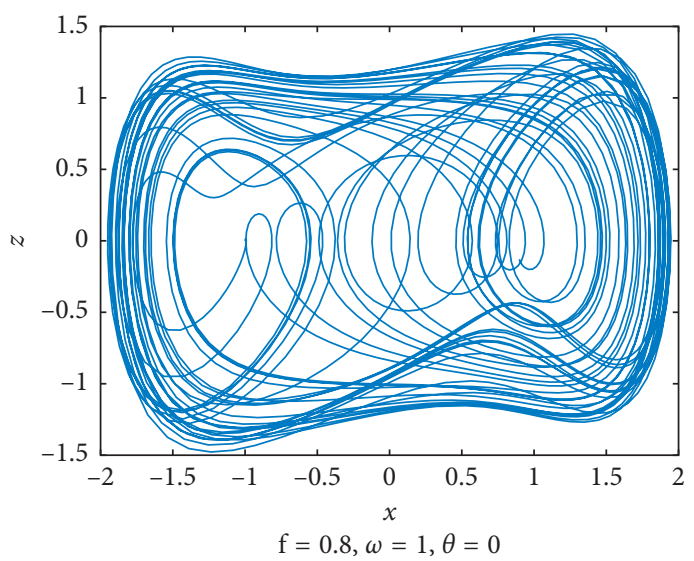

(a)

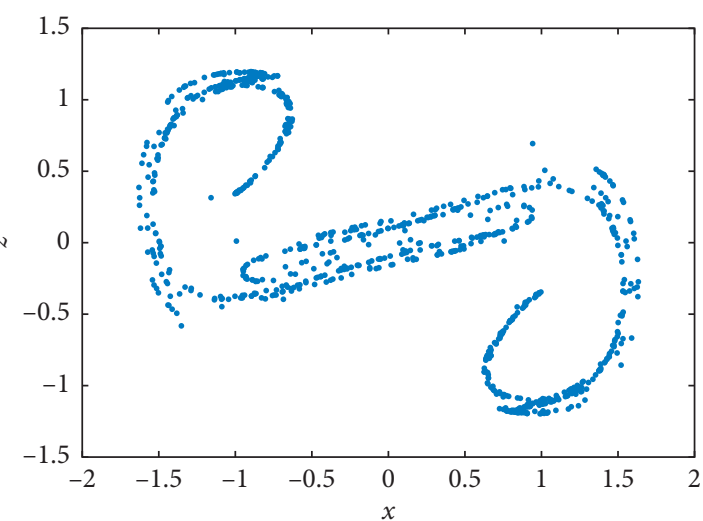

(b)

Figure 2: (a) X-y phase diagram of system (2). (b) Poincare section view of the $x-z$ plane.

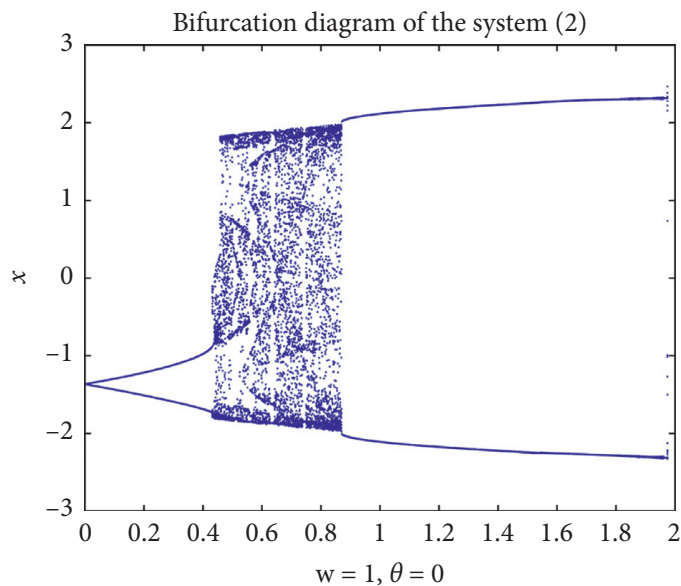

(a)

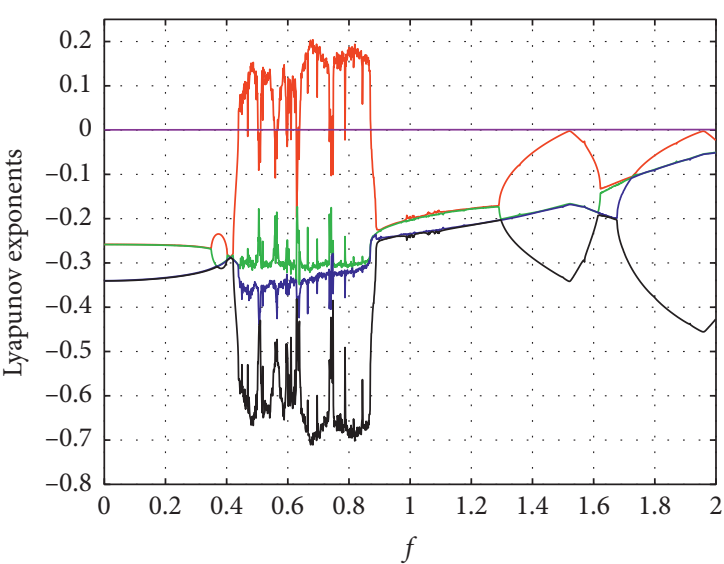

(b)

Figure 3: (a) The bifurcation diagram of (f) for system (2). (b) The lyapunov exponent diagram of (f). 
local bifurcation diagram, a chaotic state occurs in system (2) within a small range of about $\omega=0.5$, and a periodic state appears within a small range of about $\omega=1.3$.

When $\omega=1, f$ becomes 0.8 and 1, respectively. According to the above analysis, when $\omega=1$ and $f=0.8$, system (2) is in a chaotic state. When $\omega=1$ and $f=1$, system (2) is in a periodic state. In the two different kinetic states of system (2), the initial phase angle $\theta$ is made to change at an interval $[0,2 \pi]$, and the bifurcation diagram and Lyapunov exponent diagram are shown in Figure 5. As seen from the analysis in Figure 5, when system (2) is in a chaotic state, with a change to the initial phase angle $\theta$, the dynamic state of system (2) does not change. This is also the case when system (2) is in a periodic state. Therefore, the change of the initial phase angle $\theta$ of the driving force will not cause a change in the dynamic state of system (2).

\section{Basins of Attraction}

According to the set limit of system dynamics, the phase space formed by all possible initial points is divided into several disjoint subsets of the same dimension, and these subsets are called the basins of attraction. When a system with complex dynamics has multiple attractors, the boundary of the basins of attraction may have fractal characteristics. Within a certain time range, we compare the transverse maximum Lyapunov index of system (2) to the Lyapunov index of a particular track and further obtain the image of the basins of attraction for $\mathrm{x}$ $(0)-y(0)$ and $x(0)-z)(0)$ for system (2). The initial values are set to $[\mathrm{x}(0), \mathrm{y}(0), 0,0], x(0) \in[-5,5], y(0) \in[-5,5]$ and $[\mathrm{x}(0),-1, \mathrm{z}(0), 0], x(0) \in[-5,5], z(0) \in[-5,5]$, respectively. As shown in Figure 6, the basins of attraction formed by the attractor in system (2) have a complex basins structure, including both riddled basins of attraction and mixed basins of attraction. In Figure 7, the basins of attraction are featured by an overall symmetry and global mixing. In Figures 6 and 7, the red area represents the basins of attraction of the attractor at infinity, that is, the point set where the trajectory of the system diverges. The yellow area represents the basins of attraction of the chaotic attractor; that is, the overall system is stable and multiple attractors coexist. The blue area represents the transition state.

As far as current research is concerned [56, 57], the conditions for the existence of basins of attraction in an attractor are as follows:

(i) There is a smooth and invariant subspace containing chaotic attractors

(ii) There is another asymptotic final state outside the invariant subspace (not necessarily chaotic)

(iii) The horizontal Lyapunov exponent of the invariant subspace is negative

(iv) The positive finite time change is associated with the lateral stability of the unstable periodic orbit of the attractor

According to research in [58], the area of the basins of attraction will change with the initial value. The research in [5] shows that when a sieve shape appears in the basins of attraction for the Chen system, the existence of a repulsive set of positive Lebesgue measures is obtained through mathematical justification. As seen in Figure 6, the change of the basins of attraction is affected by the initial value, and the area of $|y|>2$ will show riddled basins of attraction, indicating that the attractor contains the repulsive set of the positive Lebesgue measures in the neighborhood of this area. The overall symmetry of the basins of attraction also manifests the synchronization of the system. Further research shows that it is impossible to predict the asymptotic attractor of the complex basins of attraction. The basins of attraction are shown in Figure 6; when $y$ is in the intervals $[-1.5,5]$ and $[2,5]$, the initial value of the attractor will be unpredictable. There are six attractors in a coupled system in [6], wherein the basins of attraction of two attractors are not connected, and basins of attraction of the remaining two synchronous attractors and two asynchronous attractors constitute a basin of attraction with a symmetrical mixing distribution. When $3.5>|y|>2$, there will be a yellow area not connected to the left and right, and there is also a very narrow blue area. In this paper, whether the basins of attraction of different connected states represent the basins of attraction of different attractors, the relationship between the characteristics of the sieve shape of the connected area and the state changes of coexisting attractors should be further studied. The above analysis proves that there are mathematical conditions for the existence of hidden attractors and riddled basins of attraction in system (2). We also show that the horizontal Lyapunov exponents of hidden attractors are locally unstable.

As seen from Figure 7 , the basins of attraction are a symmetrical figure and based on fractal theory are selfsimilar. According to Milnor's generalization of the classic definition of attractors, assuming that $D$ is a smooth compact manifold and bounded, $\eta$ represents the Lebesgue measure. The compact invariant set A on $D$ satisfies the following conditions:

(1) Basins of attraction $\sigma(A)=\{x: x \in d, \omega(x) \subset A\}$, with a positive measure $((A))>0$

(2) There is no set $A^{\prime}$, making $A^{\prime} A, \eta\left(\sigma\left(A^{\prime}\right)\right)-\eta(\sigma$ $(A))=0$

$\mathrm{A}$ is called an attractor. That is, when $\mathrm{A}$ has an attraction set of positive Lebesgue measures, the attractor will have an attractive neighborhood. Based on studies of attractors, if all $x \sigma(A)$ and $\delta>0$, it was discovered that when attractor $\mathrm{A}$ has riddled basins of attraction $\Phi(\mathrm{A})$, it satisfies

$$
\eta\left(\sigma_{\delta}(x) \cap \sigma(A)\right) \eta\left(\sigma_{\delta}(x) \cap \sigma(A)^{c}\right)>0,
$$

where $\sigma_{\delta}(x)$ is the $\delta$ neighborhood of $x$. As seen from this, when the basin of attraction formed by the attractor has a sieve-shaped characteristic, any neighborhood of the attractor contains the attraction set of the positive Lebesgue measures and the repulsive set of positive Lebesgue measures. In the riddled basins of attraction, there are points that are attracted by the attractor as well as points that diverge. 


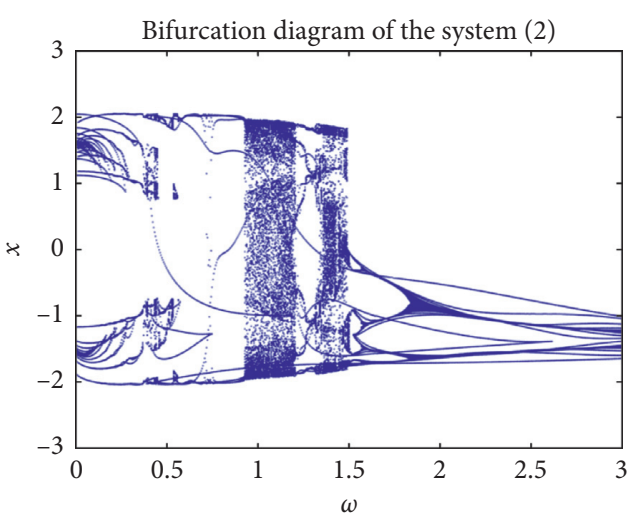

(a)

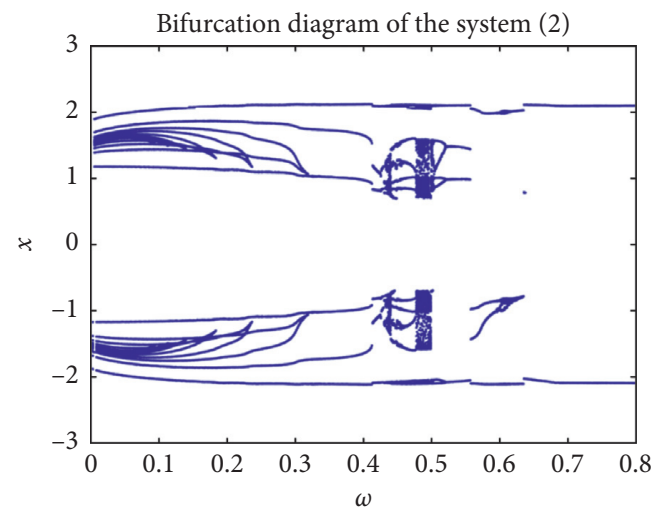

(c)

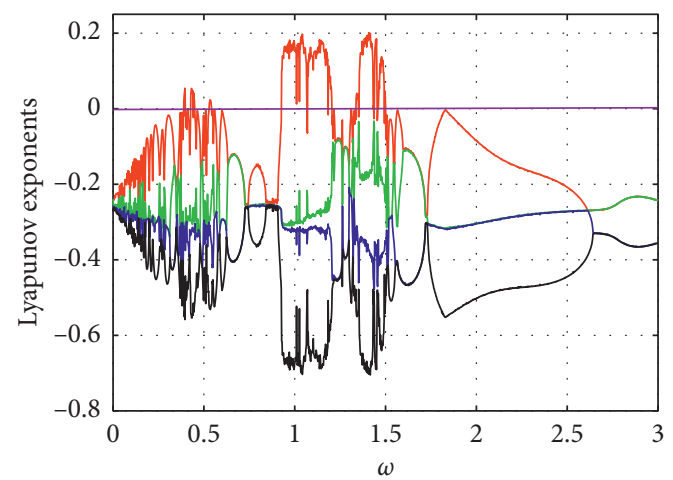

(b)

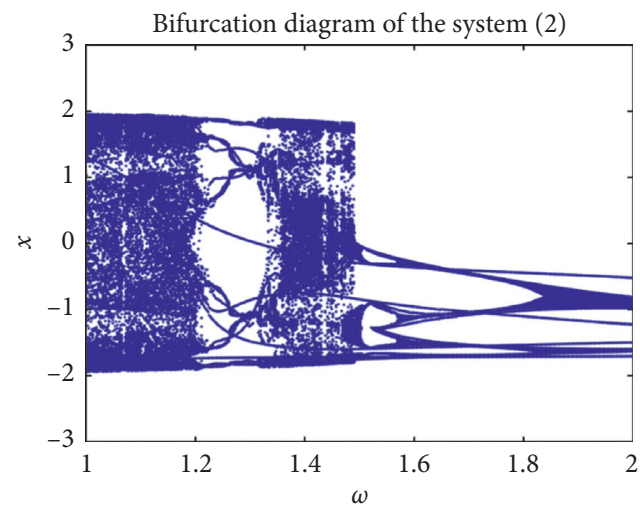

(d)

Figure 4: (a) The $\omega$ bifurcation diagram of system (2). (b) The Lyapunov exponent diagram of $\omega$. (c) The partially enlarged diagram of bifurcation of $\omega$ in the interval $[0,0.8]$. (d) The partially enlarged diagram of bifurcation of $\omega$ in the interval $[1,2]$.

Our research from [6] shows that when the system coexistence attractor exhibits synchronous changes, the attraction basins' structure is symmetrical. The research from [59] on the basins of attraction of coexistence attractors shows that when the system has multiple attractors, the basins of attraction formed by attractors will develop a mixing condition. The basins of attraction shown in Figure 7 also manifest with the characteristics of mixing and symmetry simultaneously. The study of the two subsystems in this system in this paper shows that the attractors of the two subsystems maintain synchronization between chaos periods. Based on the above references and the research in this paper, the structure of the basins of attraction in the coupled system can reflect a synchronization of the system to a certain extent. Furthermore, the structure of the attractor and the structure of the basins of attraction can also be mapped to each other under certain circumstances; that is, the symmetry of the basins of attraction can reflect the changing features of the system, and vice versa.

\section{Application of Coupled System in Weak Signal Detection}

According to the analysis in Figure 3, when the driving force frequency $\omega$ is a constant value, the state of system (2) will change from a chaotic state to a periodic state as the amplitude $f$ changes. Weak signals can be detected by using the change to system (2) state. Therefore, this paper proposes a detection system corresponding to system (2), as shown in the following equation:

$$
\left\{\begin{array}{l}
\dot{x}=z \\
\dot{y}=q \\
\dot{z}=-0.5 z+x-0.2 x^{5}-0.4(x-y)+f_{0} \cos \left(\omega_{0} t+\theta_{0}\right)+[b \cos (\omega t+\theta)+S(t)] \\
\dot{q}=-0.8\left(1-y^{2}\right) q-y-y^{3}-0.4(y-x)+f_{0} \cos \left(\omega_{0} t+\theta_{0}\right)+[b \cos (\omega t+\theta)+S(t)] .
\end{array}\right.
$$

In system (10), $b \cos (\omega t+\theta)$ represents a weak signal, and $S(t)$ represents a noise signal. $f_{0} \cos \left(\omega_{0} t+\theta_{0}\right)$ represents the driving force of the system. First, the driving force amplitude $f_{0}$ is adjusted so that the system is at a critical 


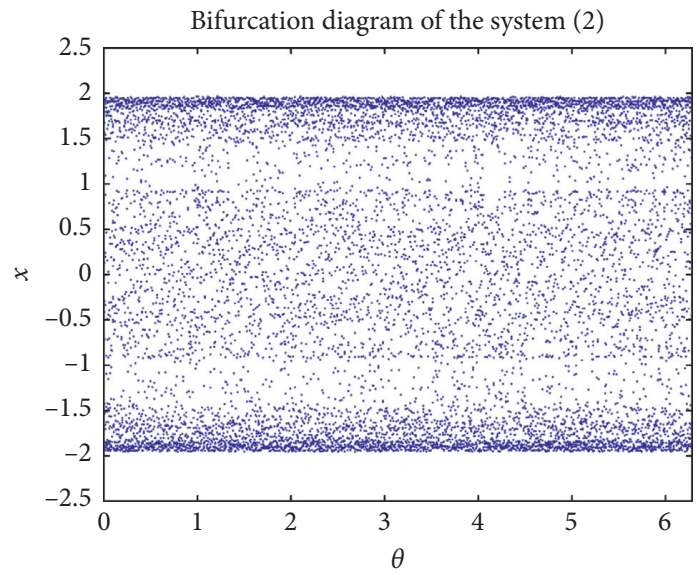

(a)

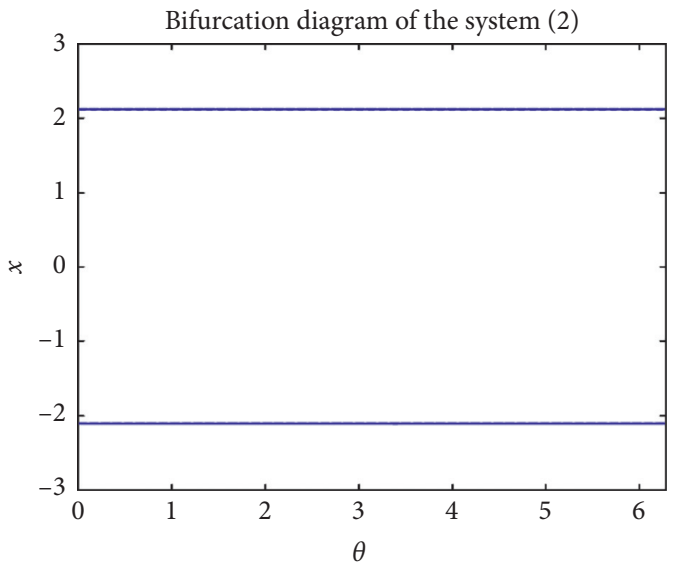

(c)

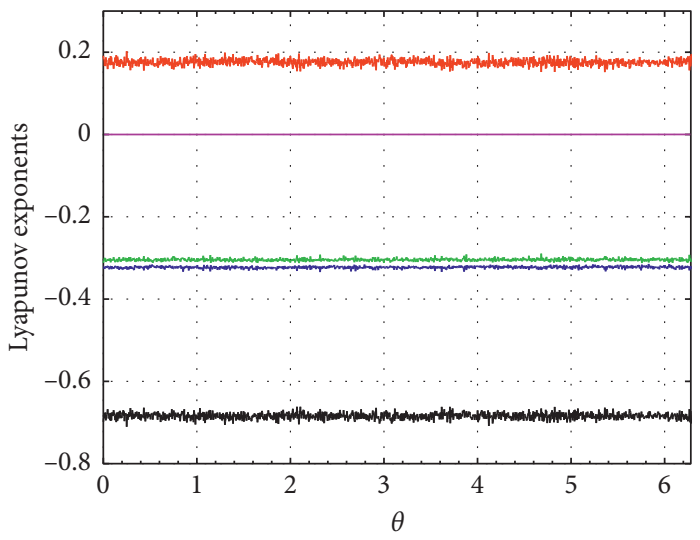

(b)

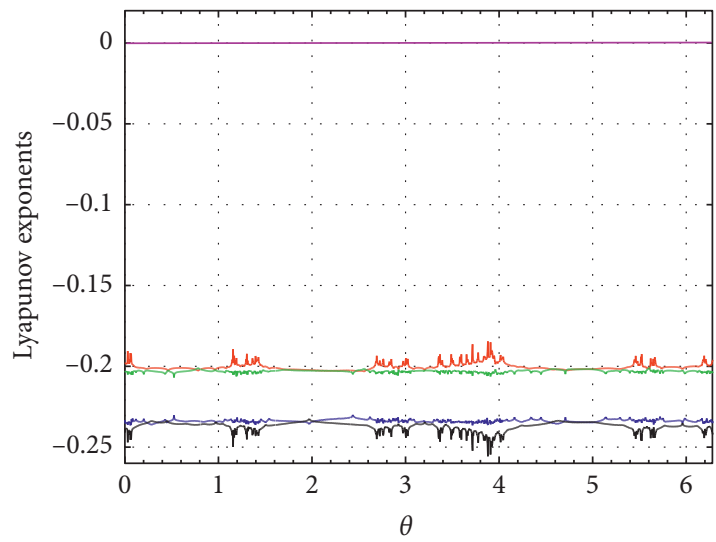

(d)

Figure 5: (a) The bifurcation diagram of $\theta$ when $f=0.8$ and $\omega=1$. (b) The Lyapunov exponent diagram of $\theta$ when $f=0.8$ and $\omega=1$. (c) The bifurcation diagram of $\theta$ when $f=1$ and $\omega=1$. (d) The Lyapunov exponent diagram of $\theta$ when $f=1$ and $\omega=1$.

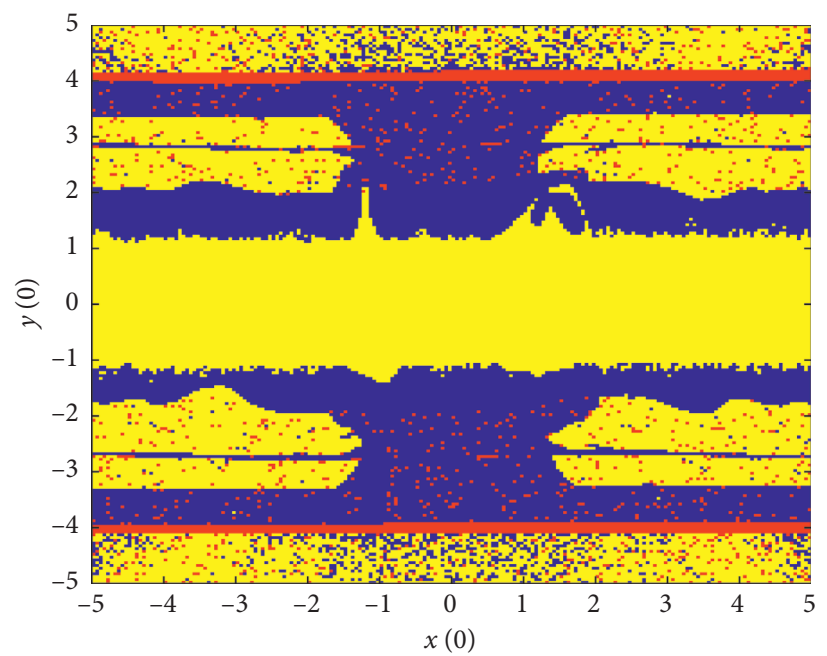

Figure 6: Diagram of $\mathrm{x}(0)-\mathrm{y}(0)$ basins of attraction for system (2). 


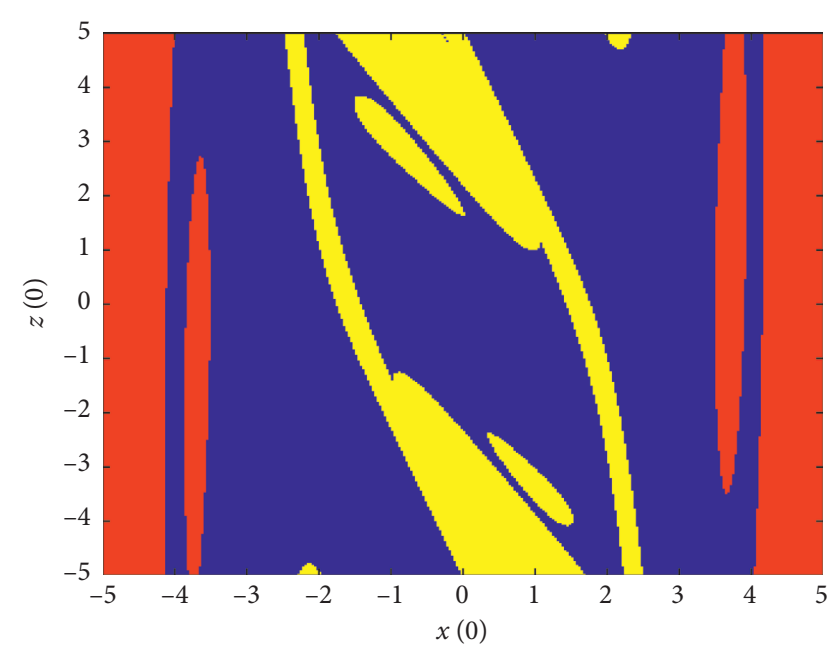

Figure 7: The $\mathrm{x}(0)-\mathrm{z}(0)$ basins of attraction diagram for system (2).

point of state transition, and, at this time, $f_{0}$ is called the system threshold. When the driving force amplitude $f_{0}=0.8747909$, system (10) is in a critical state.

5.1. Research on the Influence of Weak Signal Parameters on the System. When the frequency $\omega_{0}=1$ of system (10) is taken, the initial phase angle $\theta_{0}=0$. When amplitude $f 0=0.8747909$, Lyapunov exponent values $L_{E 1}=0.026845$, $L_{E 2}=0, \quad L_{E 3}=-0.256585, \quad L_{E 4}=-0.256435, \quad$ and $L_{E 5}=-0.508001$. At this time, the phase diagram and the power spectrum of system (10) are shown in Figure 8. Research shows that the power spectrum of the periodic signals is a discrete spectrum. The power spectrum of nonperiodic signals is a continuous spectrum. The chaotic signal is a nonperiodic signal, and the power spectrum is a continuous spectrum. Therefore, the state of system (10) may be determined based on the power spectrum. When the amplitude $f 0$ is adjusted to 0.8747909 , the phase diagram and power spectrum of system (10) are shown in Figure 8. According to Figure 8, the $\mathrm{x}-\mathrm{z}$ phase diagram of system (10) is in a chaotic state and the power spectrum is continuous; therefore, system (10) is in a chaotic state.

At this time, after a weak signal with a frequency $\omega=1$, initial phase angle $\theta=0$, and amplitude $b=1 \times 10^{-6}$ is added to system (10), Lyapunov index values of the system are $L_{E 1}=-0.014575, L_{E 2}=0, L_{E 3}=-0.251969, L_{E 4}=-0.253323$, and $L_{E 5}=-0.490388$. The phase diagram and the power spectrum for system (10) are shown in Figure 9. As seen from Figure 9, when a weak signal with an amplitude $1 \times 10^{-6}$ is added to system (10), the $x-z$ phase diagram of system (10) is in a periodic state and the power spectrum is a discrete spectrum, and the state of system (10) becomes periodic.

Because system (10) can only detect weak signals in the same or similar interval as the driving force frequency and initial phase angle, the research system can detect the frequency range of the weak signal and take the driving force frequency $\omega_{0}=1$ and the initial phase angle $\theta_{0}=0$; the initial phase angle of the weak signal $\theta=0$, and the amplitude $b=1 \times 10^{-6}$. By calculating $\omega$ in $[0.6,1.3]$, we get system $(10)$ Lyapunov exponent data when the frequency $\omega$ changes in Table 1. As seen in Table 1, when the frequency $\omega$ of the weak signal is within $[0.8,1.1]$, the dynamic state of system (10) will change.

The studied system (10) can detect the range of the initial phase angle $\theta$ of a weak signal, taking the system's driving force frequency $\omega_{0}=1$, the initial phase angle $\theta_{0}=0$, the weak signal frequency $\omega=1$, and the amplitude $b=1 \times 10^{-6}$. The driving force $f_{0}$ of the system and the weak signal $b$ are to be measured as a whole; when $\mathrm{A}$ is input into the system,

$$
\begin{aligned}
A(t)= & f_{0} \cos \left(\omega_{0} t+\theta_{0}\right)+b \cos (\omega t+\theta) \\
= & f_{0} \cos \omega_{0} t \cos \theta_{0}-f_{0} \sin \omega_{0} t \sin \theta_{0}+b \cos \omega t \cos \theta \\
& -b \sin \omega t \sin \theta .
\end{aligned}
$$

When $\omega_{0}=\omega$,

$$
\begin{aligned}
A(t)= & \left(f_{0} \cos \theta_{0}+b \cos \theta\right) \cos \omega t \\
& -\left(f_{0} \sin \theta_{0}+b \sin \theta\right) \sin \omega t \\
= & F(t) \cos (\omega t+\varphi(t)) .
\end{aligned}
$$

In equation $(10), F(t)=\sqrt{f_{0}^{2}+2 f_{0} b \cos \left(\theta_{0}-\theta\right)+b^{2}}$ $\varphi(t)=\arctan \left(\left(f_{0} \sin \theta_{0}+b \sin \theta\right) /\left(f_{0} \cos \theta_{0}+b \cos \theta\right)\right)$.

When $F(t) \geq 0.8747919$, the state of system (10) will change from a chaotic state to a periodic state. When $b=1 \times 10^{-6}$, the frequency of the driving force $\omega=1$, and the initial phase angle $\theta_{0}=1^{\circ}$ to obtain $0.91^{\circ} \leq \theta \leq=1.09^{\circ}$. As seen from the research conclusion of the initial phase angle of the driving force of system (2), the threshold of system $f 0$ does not change when the initial phase angle changes and the weak signals of different initial phase angles can be detected by adjusting the initial phase angle of the system's driving force. According to the above analysis, the system has a higher detection accuracy for the unknown weak signal, and, at the moment, the relative error of the initial phase angle measurement does not exceed \pm 0.09 .

\subsection{Research on the Antinoise Performance of System (10).} The antinoise performance of system (10) was studied by adding Gaussian white noise. When excessive noise was added, the phase diagram of system (10) converging in the periodic state will also become disorderly. Therefore, the antinoise performance of system (10) can be studied by drawing the phase diagram of system (10) under noise conditions. The regulation system is in a critical state. After Gaussian white noise power $p_{s(t)}=0.0035$ was added to system (10), the system dynamic state did not change, as shown in Figure 10(a). Then a weak signal was added to system (10), and the state of the system changes at this time, as shown in Figure 10(b). As seen in Figure 10(b), the added noise has no relevant impact on the stability of the system. Therefore, the system signal-to-noise ratio SRN is 


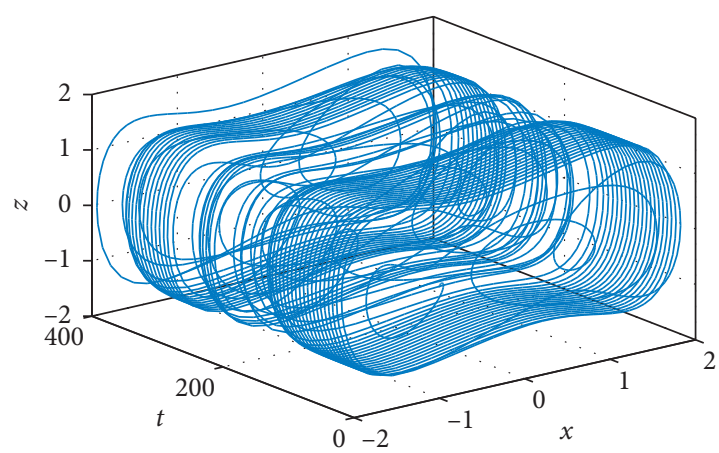

(a)

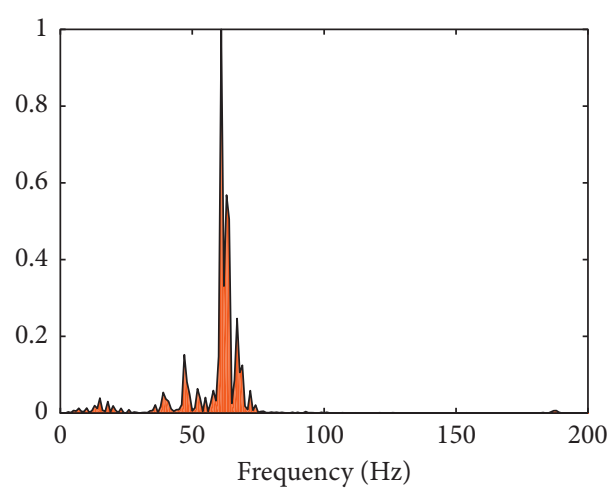

(b)

Figure 8: (a) Phase diagram of system (10) when a weak signal is not added. (b) Power spectrum diagram of system (10) when a weak signal is not added.

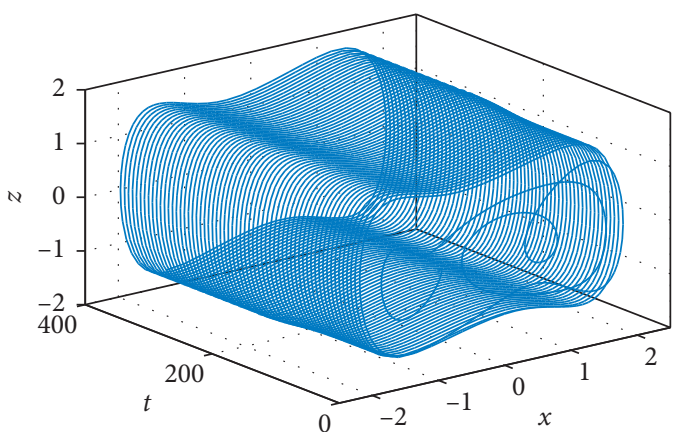

(a)

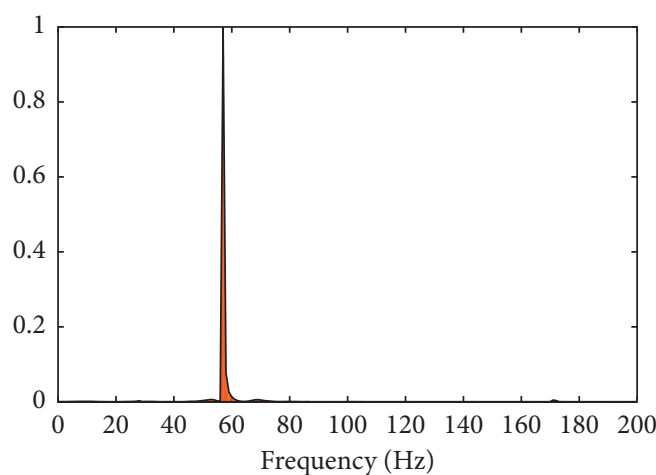

(b)

Figure 9: (a) Phase diagram of system (10) after a weak signal was added. (b) Power spectrum of system (10) after a weak signal is added.

TABLe 1: Lyapunov exponent statistics table of frequency $\omega$ withing the interval $[0.7,1.3]$.

\begin{tabular}{ccccccc}
\hline$\omega$ & $L_{E 1}$ & $L_{E 2}$ & $L_{E 3}$ & $L_{E 4}$ & $L_{E 5}$ & System state \\
\hline 0.6 & 0.054782 & 0 & -0.253654 & -0.264728 & -0.542015 & Chaotic state \\
0.7 & 0.001082 & 0 & -0.253727 & -0.259955 & -0.506094 & Chaotic state \\
0.8 & 0 & -0.010830 & -0.254174 & -0.253927 & -0.493878 & Periodic state \\
0.9 & 0 & -0.025991 & -0.250020 & -0.251349 & -0.478236 & Periodic state \\
1.1 & 0 & -0.029113 & -0.251380 & -0.250912 & -0.474047 & Periodic state \\
1.2 & 0.069098 & 0 & -0.272891 & -0.274010 & -0.577603 & Chaotic state \\
1.3 & 0.015809 & 0 & -0.259136 & -0.261232 & -0.521500 & Chaotic state \\
\hline
\end{tabular}

$$
\begin{aligned}
S R N & =10 \lg \left(\frac{p_{b}}{p_{S(t)}}\right) \\
& =10 \lg \left[0.5 \frac{\left(1 \times 10^{-6}\right)^{2}}{(0.0035)^{2}}\right] \approx-73.892 \mathrm{~dB}
\end{aligned}
$$

In [53], only a signal with a size of 0.01 can be detected and the signal-to-noise ratio can only reach $-45.85 \mathrm{~dB}$. The system in [54] can detect signals with the size of $1 \times 10^{-9}$, but the noise signal that the system can be immune to can only reach a size of $1 \times 10^{-9}$. The system in [55] can detect signals of a larger frequency range, but the fluctuation range of the initial phase angle from the measured signal is too large, and the error range is \pm 60.6 , which is not conducive to confirmation of signal parameters. The system proposed in this paper can detect signals sized $1 \times 10^{-6}$, and the signal-tonoise ratio reaches $-73.892 \mathrm{~dB}$. Moreover, the immune signal reached the order of $3.5 \times 10^{-3}$, and the error range of the initial phase angle is \pm 0.09 . In summary, the system introduced in this paper has superior detection performance.

\section{Circuit Design and Realization of System}

6.1. Circuit Design of System. According to the circuit principle and the characteristics of electronic components, 


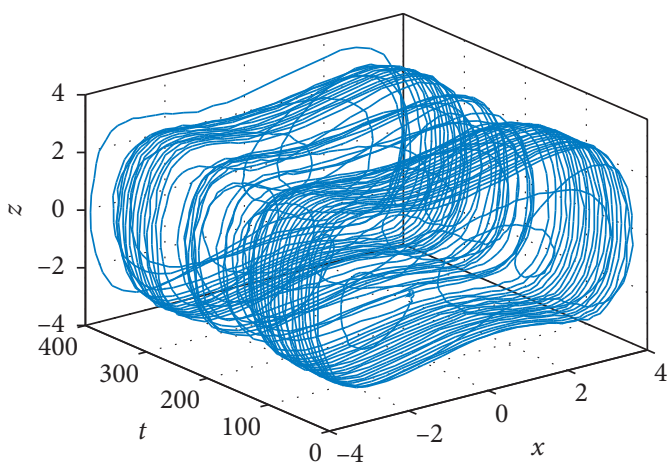

(a)

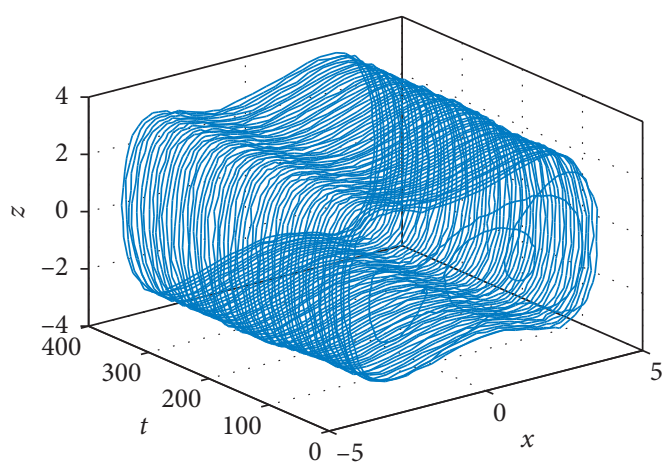

(b)

Figure 10: (a) Phase diagram of system (10) after adding Gaussian white noise. (b) Phase diagram of system (10) after adding a weak signal in the presence of Gaussian white noise.
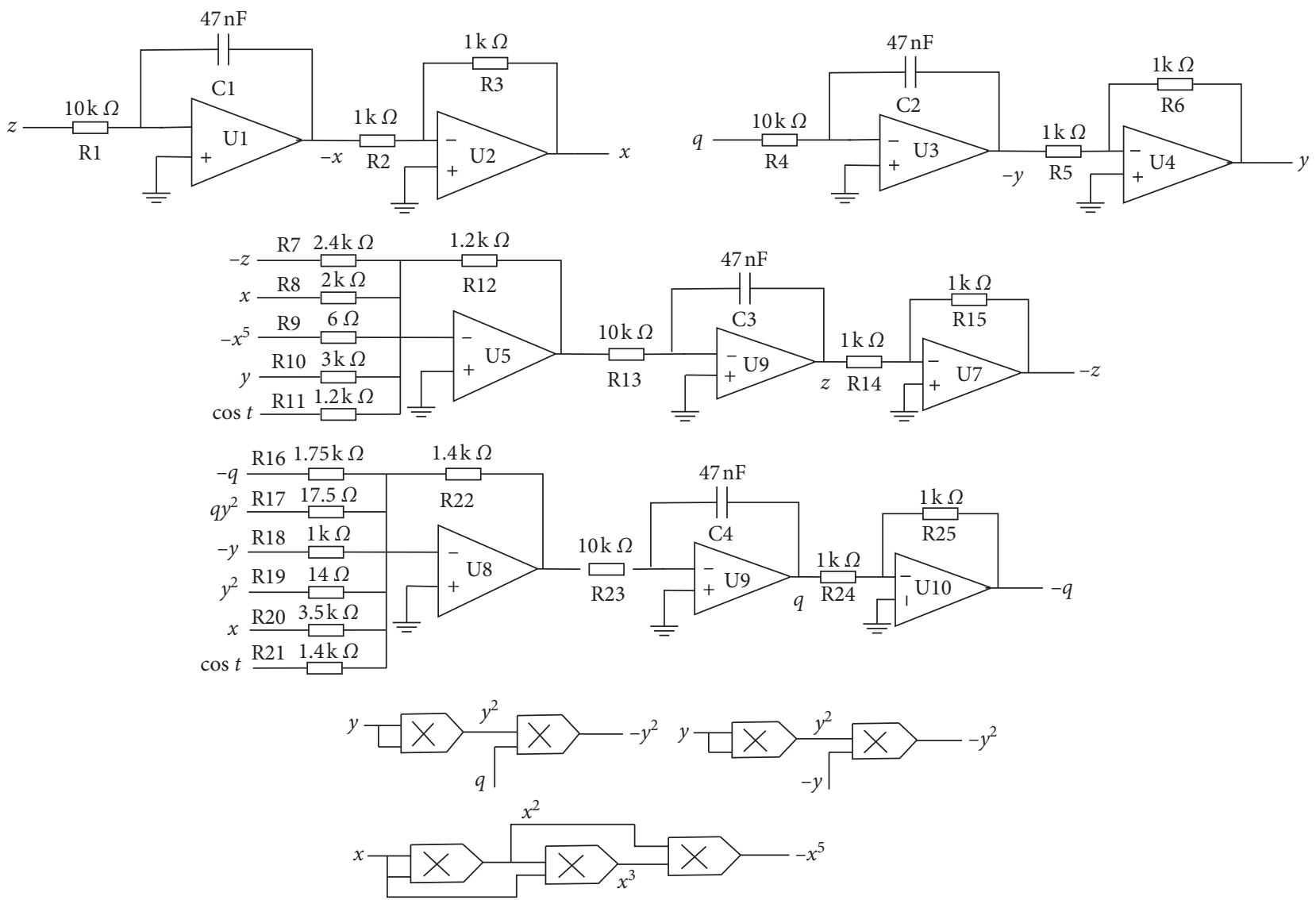

Figure 11: Circuit diagram schematic for system (2). 


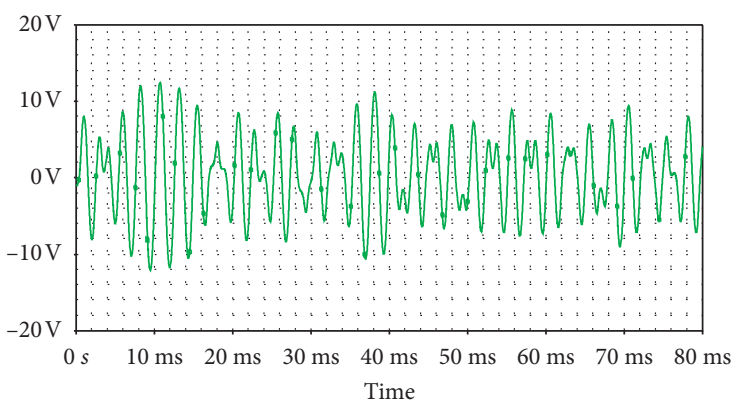

$\square \mathrm{V}(x)$

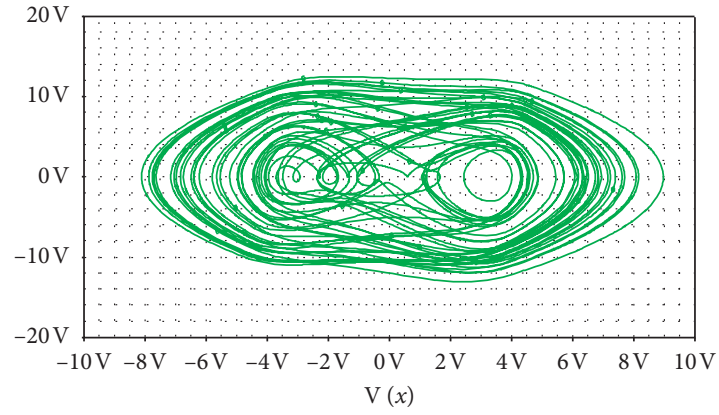

$\square \mathrm{V}(z)$

(a)

(b)

Figure 12: (a) Oscillogram of system (2) variable x; (b) x-z phase diagram of system (2).

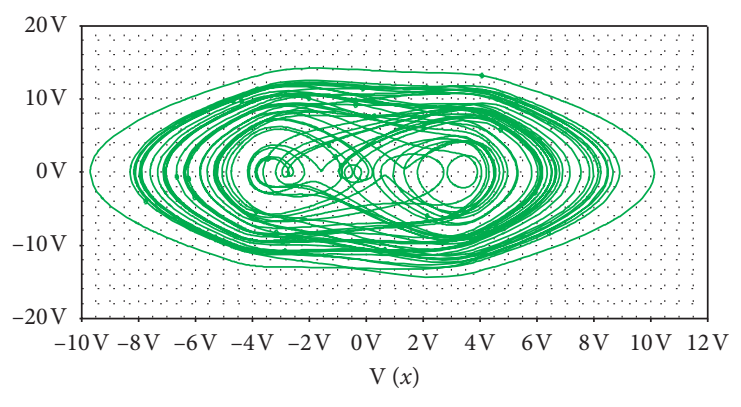

$\square \mathrm{V}(z)$

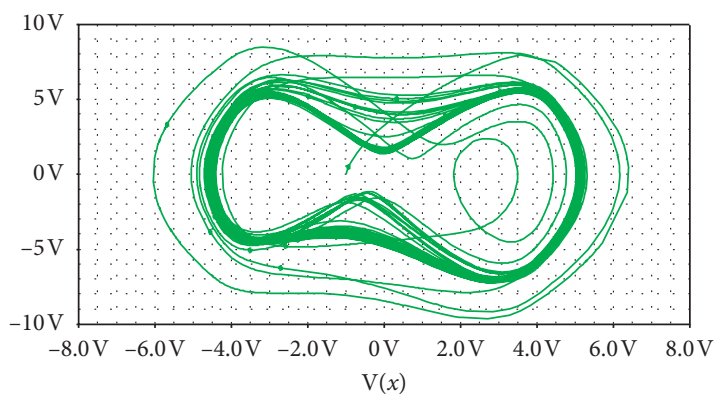

$\square \mathrm{V}(z)$

(a)

(b)

FIgURE 13: (a) x-z phase diagram of (10) without a weak signal; (b) x-z phase diagram of system (10) with a weak signal.

the circuit equation (14) as shown in system (2) can be obtained as follows:

$$
\left\{\begin{array}{l}
\frac{d V_{x}}{d t}=-\frac{1}{R_{1} C_{1}} V_{z} \\
\frac{d V_{y}}{d t}=-\frac{1}{R_{2} C_{2}} V_{q}, \\
\frac{d V_{z}}{d t}=-\frac{1}{R_{13} C_{3}}\left(-\frac{R_{12}}{R_{7}} V_{z}+\frac{R_{12}}{R_{8}} V_{x}-\frac{R_{12}}{R_{9}} V_{x^{5}}+\frac{R_{12}}{R_{10}} V_{y}+\frac{R_{12}}{R_{11}} V_{\cos t}\right) \\
\frac{d V_{q}}{d t}=-\frac{1}{R_{23} C_{4}}\left(-\frac{R_{22}}{R_{16}} V_{q}+\frac{R_{22}}{R_{17}} V_{q y^{2}}-\frac{R_{22}}{R_{18}} V_{y}-\frac{R_{22}}{R_{19}} V_{y^{3}}+\frac{R_{22}}{R_{20}} V_{x}+\frac{R_{22}}{R_{21}} V_{\cos t}\right)
\end{array}\right.
$$

The circuit for equation (14) is shown in Figure 11. System (2) is compared with equation (14) to make their coefficients equal, and the selected device parameters $C_{1}=C_{2}=C_{3}=C_{4}=47 \mathrm{nF}, R_{2}=R_{3}=R_{5}=R_{6}=R_{14}=R_{15}$
$=R_{18}=R_{24}=R_{25}=1 \mathrm{k} \Omega, R_{1}=R_{4}=R_{13}=R_{23}=10 \mathrm{k} \Omega, R_{11}$ $=R_{12}=1.2 \mathrm{k} \Omega, R_{21}=R_{22}=1.4 \mathrm{k} \Omega, R_{7}=2.4 \mathrm{k} \Omega, R_{8}=2 \mathrm{k} \Omega$, $R_{9}=6 \Omega, \quad R_{10}=3 k \Omega, \quad R_{16}=1.75 \mathrm{~K} \Omega, \quad R_{17}=17.5 \Omega$, $R_{19}=14 \Omega$, and $R_{20}=3.5 \mathrm{k} \Omega$. The operational amplifier 


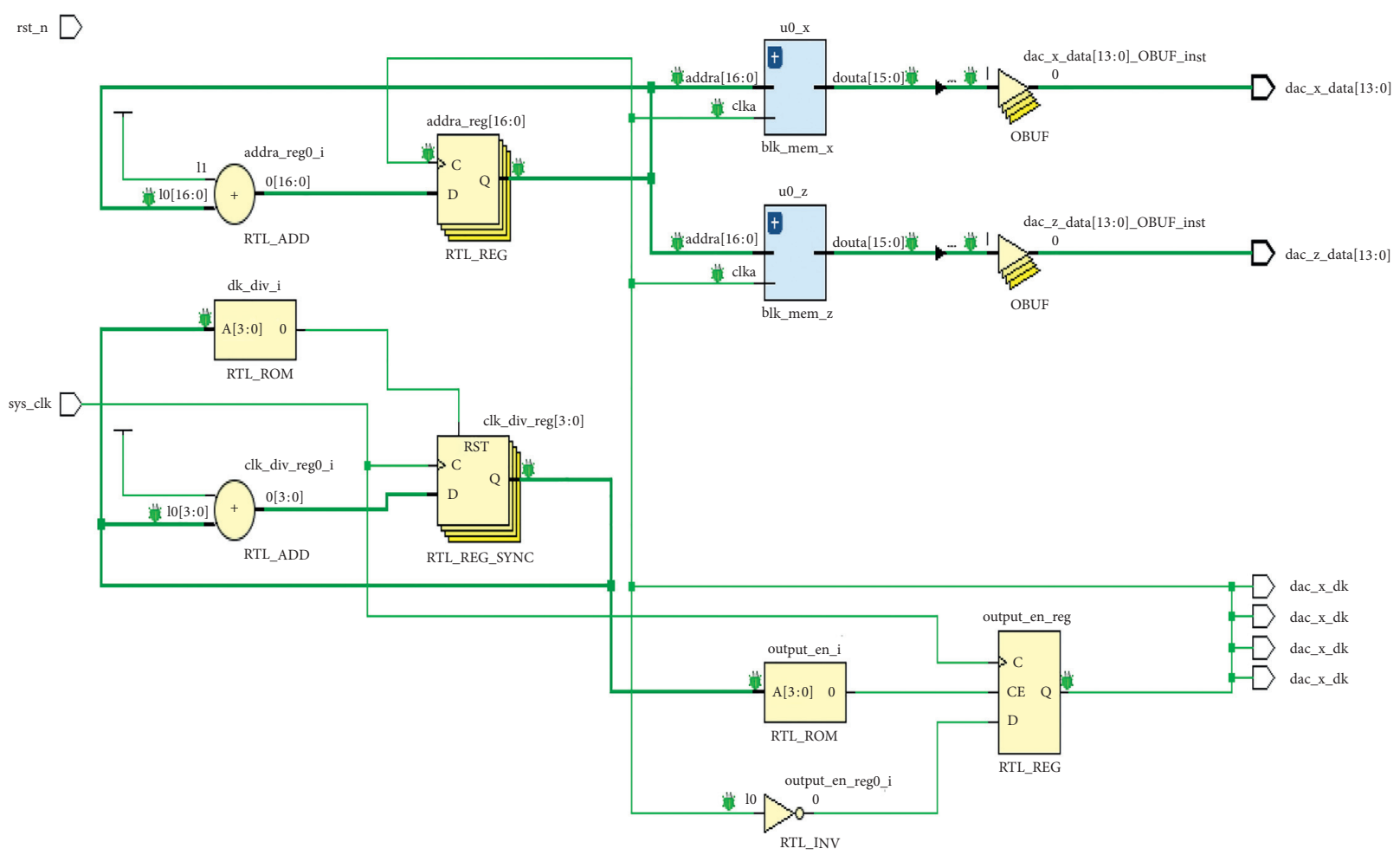

FIgURE 14: RTL view based on FPGA implementation.

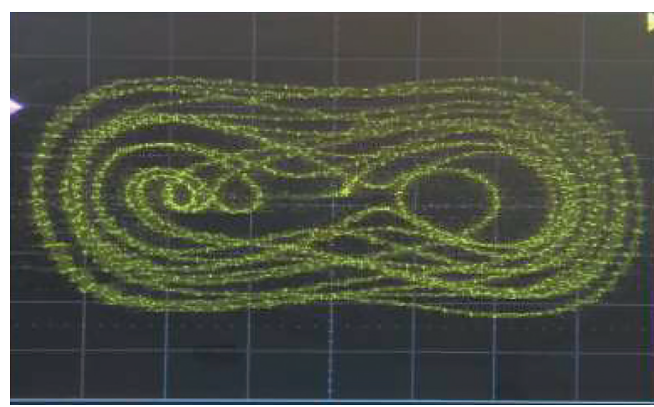

(a)

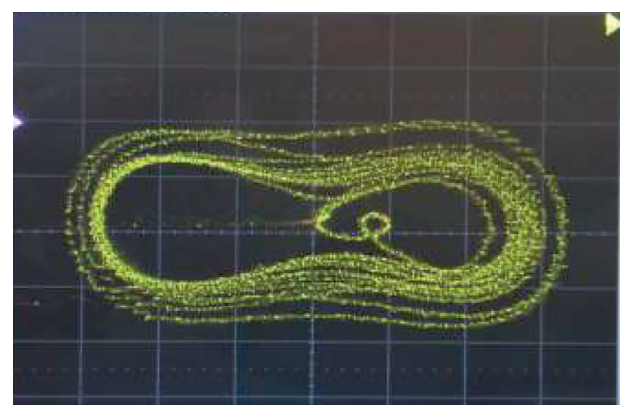

(b)

FIGURE 15: (a) X-z phase diagram of system (10) before adding a weak signal; (b) X-z phase diagram of system (10) after adding a weak signal.

model selected by the circuit is TL082, and the multiplier model is AD633.

\subsection{System Circuit Simulation and FPGA Implementation.} In this paper, OrCAD was used to simulate the system circuit, the amplitude was set as $f=8 \mathrm{~V}$, and the frequency $\varphi=400 \mathrm{~Hz}$, with the waveform diagram of the state variable $x$ and the phase diagram of $\mathrm{x}-\mathrm{z}$ shown in Figure 12 .

Test the detection performance of system (10), take the frequency $\varphi=400 \mathrm{~Hz}$, and adjust the amplitude $f=13.5664362 \mathrm{~V}$, with system (10) in a chaotic state as shown in Figure 13(a). When the driving force amplitude $f=13.5664372$ is adjusted, the state of system (10) changes to a periodic state, as shown in Figure 13(b). Therefore, system (10) can effectively detect the sine signal with an amplitude of $1 \times 10^{-6} \mathrm{~V}$.
Based on FPGA technology and fixed-point number method, hardware experiment is carried out on system (10). We use Xilinx zynq-7000 XC7Z020 FPGA chip and AD9767 dual-port parallel 14-bit A/D module with the highest conversion rate of $125 \mathrm{MHz}$. The software used is Vivado 17.4 and system generator compiler is used to realize the joint debugging of MATLAB and FPGA. In addition, we also use SIGLENT 1202X digital oscilloscope to visualize analog output. After compiling, the automatically generated RTL view is shown in Figure 14.

The regulation system was in a critical state, as shown in Figure 15(a). After adding a weak signal, the phase diagram of system (10) is shown in Figure 15(b), and the state of system (10) changes. Therefore, system (10) can effectively detect weak signals. 


\section{Conclusion}

Through calculation, this paper concludes that system (2) has an infinite number of equilibrium points, and there is a Lyapunov exponent greater than 0 . Therefore, system (2) is a hidden attractor coupled chaotic system. The analysis of the basins of attraction shows that system (2) has the characteristics of structural symmetry, and the state of the attractor changes synchronously. Further research finds that the Lyapunov exponent of the hidden attractor is locally unstable. The research on the Lyapunov indexes of all parameters of system (10) driving force has proved that system (10) can more accurately detect signals with a size of $1 \times 10^{-6}$, and the research on system (10) with noise shows that system (10) has excellent antinoise performance. Finally, circuit simulation and FPGA implementation have demonstrated the theoretical analysis and the accuracy of weak signal detection to be correct.

In future research, the focus will be on how to detect different types of signals, how to improve the system's antinoise performance, and identifying the system's state. As there is no unified conclusion on the structure of the basins of attraction, the research results should be further verified. Moreover, no unified results have been achieved in the theoretical research on the structure of the basins of attraction, and thus the research on the basins of attraction will also be a hot spot in the future.

\section{Data Availability}

The data used to support the findings of this study are included within the article.

\section{Conflicts of Interest}

The authors declare that they have no conflicts of interest.

\section{Acknowledgments}

This work was supported by the National Natural Science Foundation of China under Grants 61504013 and 61561022, the Natural Science Foundation of Hunan Province under Grants 2019JJ50648, 2020JJ4315, and 2017JJ3254, and $\mathrm{Hu}-$ nan Provincial Education Department under Grant 20B216.

\section{References}

[1] E. N. Lorenz, "Deterministic nonperiodic flow," Journal of the Atmospheric Sciences, vol. 20, no. 2, pp. 130-141, 1963.

[2] Z. Liu, Y. Li, and G. Chen, "The basin of attraction of the Chen attractor," Chaos, Solitons \& Fractals, vol. 34, no. 5, pp. 1696-1703, 2007.

[3] S. P. Raj, S. Rajasekar, and K. Murali, "Coexisting chaotic attractors, their basin of attractions and synchronization of chaos in two coupled Duffing oscillators," Physics Letters A, vol. 264, no. 4, pp. 283-288, 1999.

[4] J. A. Wright, J. H. B. Deane, M. Bartuccelli et al., "Basins of attraction in forced systems with time-varying dissipation," Communications in Nonlinear Science and Numerical Simulation, vol. 29, no. 1-3, pp. 72-87, 2015.
[5] G. Wang, F. Yuan, G. Chen et al., "Coexisting multiple attractors and riddled basins of a memristive system," Chaos: An Interdisciplinary Journal of Nonlinear Science, vol. 28, no. 1, Article ID 013125, 2018.

[6] A. Bayani, K. Rajagopal, A. J. M. Khalaf, S. Jafari, G. D. Leutcho, and J. Kengne, "Dynamical analysis of a new multistable chaotic system with hidden attractor: antimonotonicity, coexisting multiple attractors, and offset boosting," Physics Letters A, vol. 383, no. 13, pp. 1450-1456, 2019.

[7] L. Xiong, S. Zhang, Y. Zeng, and B. Liu, "Dynamics of a new composite four-Scroll chaotic system," Chinese Journal of Physics, vol. 56, no. 5, pp. 2381-2394, 2018.

[8] H. Jahanshahi, A. Yousefpour, J. M. Munoz-Pacheco, I. Moroz, Z. Wei, and O. Castillo, "A new multi-stable fractional-order four-dimensional system with self-excited and hidden chaotic attractors: dynamic analysis and adaptive synchronization using a novel fuzzy adaptive sliding mode control method," Applied Soft Computing, vol. 87, p. 105943, 2020.

[9] Q. Deng, C. Wang, and L. Yang, "Four-wing hidden attractors with one stable equilibrium point," International Journal of Bifurcation and Chaos, vol. 30, no. 06, p. 2050086, 2020.

[10] Sundarapandian Vaidyanathan, Aceng Sambas, Mustafa Mamat, and W. S. Mada Sanjaya, "A new three-dimensional chaotic system with a hidden attractor, circuit design and application in wireless mobile robot," Archives of Control Sciences, vol. 27, no. LXIII, pp. 541-554, 2017.

[11] Yu Fei, Li Liu, H. Shen et al., "Dynamic analysis, Circuit design and Synchronization of a novel 6D memristiv fourwing hyperchaotic system with multiple coexisting attractors," Complexity, vol. 2020, Article ID 5904607, , 2020.

[12] F. Yu, L. Liu, H. Shen et al., "Multistability analysis, coexisting multiple attractors and FPGA implementation of Yu-Wang four-wing chaotic system," Mathematical Problems in Engineering, vol. 2020, Article ID 7530976, , 2020.

[13] A. Sambas, S. Vaidyanathan, E Tlelo-Cuautle et al., "A novel chaotic system with two circles of equilibrium points: multistability, electronic circuit and FPGA realization," Electronics, vol. 8, no. 11, Article ID 1211, 2019.

[14] C. Wang, H. Xia, and L. Zhou, "A memristive hyperchaotic multiscroll jerk system with controllable scroll numbers," International Journal of Bifurcation and Chaos, vol. 27, no. 06, Article ID 1750091, 2017.

[15] R. Wu and C. Wang, "A new simple chaotic circuit based on memristor," International Journal of Bifurcation and Chaos, vol. 26, no. 09, Article ID 1650145, 2016.

[16] C. Wang, X. Hu, and L. Zhou, "Implementation of a new memristor-based multiscroll hyperchaotic system," Pramana, vol. 88, no. 2, p. 34, 2017.

[17] H. Lin, C. Wang, W. Yao, and Y. Tan, "Chaotic dynamics in a neural network with different types of external stimuli," Communications in Nonlinear Science and Numerical Simulation, vol. 90, Article ID 105390, 2020.

[18] W. Yao, C. Wang, Y. Sun, C. Zhou, and H. Lin, "Exponential multistability of memristive Cohen-Grossberg neural networks with stochastic parameter perturbations," Applied Mathematics and Computation, vol. 386, p. 125483, 2020.

[19] H. Lin, C. Wang, and Y. Tan, "Hidden extreme multistability with hyperchaos and transient chaos in a Hopfield neural network affected by electromagnetic radiation," Nonlinear Dynamics, vol. 99, pp. 2369-2386, 2020. 
[20] V. T. Pham, D. S. Ali, N. M. G. Al-Saidi, K. Rajagopal, F. E. Alsaadi, and S. Jafari, "A novel mega-stable chaotic circuit," Radioengineering, vol. 29, no. 1, pp. 140-146, 2020.

[21] V. -T. Pham, S. Vaidyanathan, C. Volos, S. Jafari, and T. Kapitaniak, "A new multi-stable chaotic hyperjerk system, its special features, circuit realization, control and synchronization," Archives of Control Sciences, vol. 30, no. 1, pp. 23-45, 2020.

[22] J. Jin and L. Cui, "Fully integrated memristor and its application on the scroll-controllable hyperchaotic system," Complexity, vol. 2019, Article ID 4106398, 2020.

[23] J. Jin, "Programmable multi-direction fully integrated chaotic oscillator,” Microelectronics Journal, vol. 75, pp. 27-34, 2018.

[24] J. Jin and L. Zhao, "Low voltage low power fully integrated chaos generator," Journal of Circuits, Systems and Computers, vol. 27, no. 10, Article ID 1850155, 2018.

[25] J. Sun, Mu Peng, F. Liu, and C. Tang, "Protecting compressive ghost imaging with hyper-chaotic system and DNA encoding," Complexity, vol. 2020, Article ID 8815315, 2020.

[26] C. Xu, J. Sun, and C. Wang, "A novel image encryption algorithm based on bit-plane matrix rotation and hyper chaotic systems," Multimedia Tools and Applications, vol. 79, no. 9-10, pp. 5573-5593, 2020.

[27] Xi Chen, S. Qian, Yu Fei et al., "Pseudorandom number generator based on three kinds of four-wing memristive hyperchaotic system and its application in image encryption," Complexity, vol. 2020, Article ID 8274685, 2020.

[28] S. Wang, C. Wang, and C. Xu, "An image encryption algorithm based on a hidden attractor chaos system and the Knuth-Durstenfeld algorithm," Optics and Lasers in Engineering, vol. 128, p. 105995, 2020.

[29] A. Sambas, S. Vaidyanathan, E. Tlelo-Cuautle et al., "A 3-D multi-stable system with a peanut-shaped equilibrium curve: circuit design, FPGA realization, and an application to image encryption," The Institute of Electrical and Electronics Engineers Access, vol. 8, pp. 137116-137132, 2020.

[30] Y. Huang, L. Huang, Y. Wang, Y. Peng, and F. Yu, "Shape synchronization in driver-response of 4 -D chaotic system and its application in image encryption," The Institute of Electrical and Electronics Engineers Access, vol. 8, pp. 135308-135319, 2020.

[31] L. Zhou, F. Tan, and F. Yu, "A robust synchronization-based chaotic secure communication scheme with double-layered and multiple hybrid networks," The Institute of Electrical and Electronics Engineers Systems Journal, vol. 14, no. 2, pp. 2508-2519, 2020.

[32] L. Zhou, F. Tan, F. Yu, and W. Liu, "Cluster synchronization of two-layer nonlinearly coupled multiplex networks with multi-links and time-delays," Neurocomputing, vol. 359, pp. 264-275, 2019.

[33] F. Peng, X. Zhu, and M. Long, "An ROI privacy protection scheme for H. 264 video based on FMO and chaos," The Institute of Electrical and Electronics Engineers Transactions on Information Forensics and Security, vol. 8, no. 10, pp. 16881699, 2013.

[34] F. Yu, S. Qian, X. Chen et al., "A new 4D four-wing memristive hyperchaotic system: dynamical analysis, electronic circuit design, shape synchronization and secure communication," International Journal of Bifurcation and Chaos, vol. 30, no. 10, Article ID 2050147, 2020.

[35] J. Jin, L. Zhao, M. Li, F. Yu, and Z. Xi, "Improved zeroing neural networks for finite time solving nonlinear equations," Neural Computing and Applications, vol. 32, no. 9, pp. 4151-4160, 2020.
[36] F. Yu, L. Liu, L. Xiao, K. Li, and S. Cai, "A robust and fixedtime zeroing neural dynamics for computing time-variant nonlinear equation using a novel nonlinear activation function," Neurocomputing, vol. 350, pp. 108-116, 2019.

[37] J. Jin, “A robust zeroing neural network for solving dynamic nonlinear equations and its application to kinematic control of mobile manipulator," Complex \& Intelligent Systems, 2020.

[38] J. Jin and J. Gong, "An interference-tolerant fast convergence zeroing neural network for dynamic matrix inversion and its application to mobile manipulator path tracking," Alexandria Engineering Journal, 2020.

[39] F. Yu, L. Liu, S. Qian et al., "Chaos-based application of a novel multistable 5D memristive hyperchaotic system with coexisting multiple attractors," Complexity, vol. 2020, Article ID 8034196, 2020.

[40] F. Yu, Q. Wan, J. Jin et al., "Design and FPGA implementation of a pseudorandom number generator based on a four-wing memristive hyperchaotic system and Bernoulli map," The Institute of Electrical and Electronics Engineers Access, vol. 7, pp. 181884-181898, 2019.

[41] F. Yu, L. Li, Q. Tang et al., "A survey on true random number generators based on chaos," Discrete Dynamics in Nature and Society, vol. 2019, Article ID 2545123, 2019.

[42] J. Yan and L. Lu, "Improved Hilbert-Huang transform based weak signal detection methodology and its application on incipient fault diagnosis and ECG signal analysis," Signal Processing, vol. 98, pp. 74-87, 2014.

[43] Y. Wang, G. Xu, L. Liang, and K. Jiang, "Detection of weak transient signals based on wavelet packet transform and manifold learning for rolling element bearing fault diagnosis," Mechanical Systems and Signal Processing, vol. 54-55, pp. 259-276, 2015.

[44] L. Lu, J. Yan, and C. W. de Silva, "Dominant feature selection for the fault diagnosis of rotary machines using modified genetic algorithm and empirical mode decomposition," Journal of Sound and Vibration, vol. 344, pp. 464-483, 2015.

[45] D. L. Birx and S. J. Pipenberg, "Chaotic oscillators and complex mapping feed forward networks (CMFFNS) for signal detection in noisy environments," The Institute of Electrical and Electronics Engineers in Proceedings 1992] IJCNN International Joint Conference on Neural Networks, vol. 2, pp. 881-888, Baltimore, MD, USA, January 1992.

[46] Z.-H. Lai and Y.-G. Leng, "Weak-signal detection based on the stochastic resonance of bistable Duffing oscillator and its application in incipient fault diagnosis," Mechanical Systems and Signal Processing, vol. 81, pp. 60-74, 2016.

[47] Y. Jiang, H. Zhu, and Z. Li, “A new compound faults detection method for rolling bearings based on empirical wavelet transform and chaotic oscillator," Chaos, Solitons \& Fractals, vol. 89, pp. 8-19, 2016.

[48] X. Liu and X. Liu, "Weak signal detection research based on duffing oscillator used for downhole communication," Journal of Computers, vol. 6, no. 2, pp. 359-367, 2011.

[49] F. Yang, L. Jing, W. Zhang, Y. Yan, and H. Ma, "Experimental and numerical studies of the oblique defects in the pipes using a chaotic oscillator based on ultrasonic guided waves," Journal of Sound and Vibration, vol. 347, pp. 218-231, 2015.

[50] P. Kumar, S. Narayanan, and S. Gupta, "Investigations on the bifurcation of a noisy Duffing-van der Pol oscillator," Probabilistic Engineering Mechanics, vol. 45, pp. 70-86, 2016.

[51] N. Chunyan and S. Yaowu, "The research of Chaotic characteristic identification base on weak signal detection," ACTA Metrological Sinica, vol. 10, no. 4, pp. 308-313, 2000. 
[52] T. Xie, X. Wei, and R. Yu, "Noise immunity analysis in external excitation chaotic oscillator detecting system[C]//2010 International Conference on Intelligent System Design and Engineering Application," The Institute of Electrical and Electronics Engineers, vol. 1, pp. 1013-1016, 2010.

[53] Z. Zhao and S. Yang, "Application of vanderPol-Duffing oscillator in weak signal Detection," Computers and Electrical Engineering, vol. 41, pp. 1-8, 2015.

[54] A. Gokyildirim, Y. Uyaroglu, and I. Pehlivan, "A novel chaotic attractor and its weak signal detection application," Optik, vol. 127, no. 19, pp. 7889-7895, 2016.

[55] Y. Zhang, H. Mao, H. Mao, and Z. Huang, "Detection the nonlinear ultrasonic signals based on modified Duffing equations," Results in Physics, vol. 7, pp. 3243-3250, 2017.

[56] U. Chaudhuri and A. Prasad, "Complicated basins and the phenomenon of amplitude death in coupled hidden attractors," Physics Letters A, vol. 378, no. 9, pp. 713-718, 2014.

[57] S. Camargo, R. L. Viana, and C. Anteneodo, "Intermingled basins in coupled Lorenz systems," Physical Review E, vol. 85, no. 3, Article ID 036207, 2012

[58] B. L. Lan and C. Yapp, "Dissipative relativistic standard map: periodic attractors and basins of attraction," Chaos, Solitons \& Fractals, vol. 37, no. 5, pp. 1300-1304, 2008.

[59] C. Chen, J. Chen, H. Bao, M. Chen, and B. Bao, "Coexisting multi-stable patterns in memristor synapse-coupled Hopfield neural network with two neurons," Nonlinear Dynamics, vol. 95, no. 4, pp. 3385-3399, 2019. 